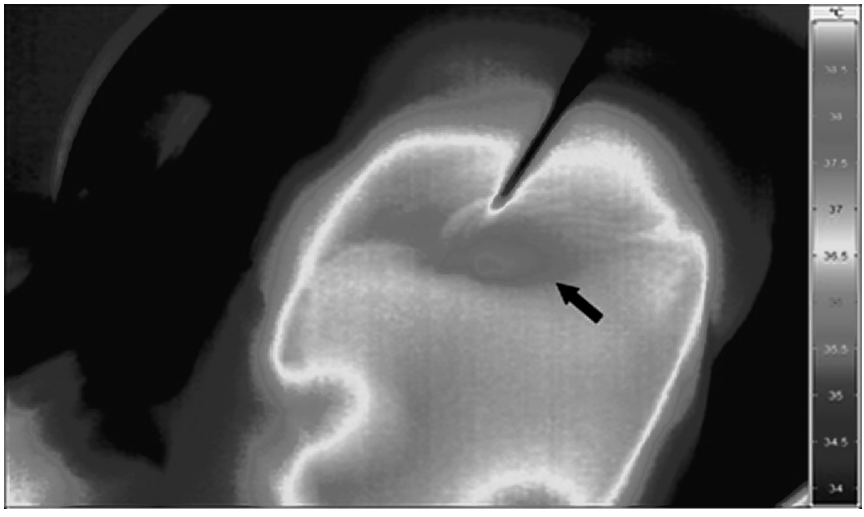

Abstract S89 Figure 1 Infra-red thermal imaging of coil apparatus with mouse in situ receiving hyperthermia treatment (arrow indicates tumour).

Ferucarbotranfor labelling. Subcutaneous tumours were induced by co-injecting 5 million OVCAR cells and 0.5 million SPION-labelled MSCs.

- Images of cells within mice were obtained on a 9.4Thorizontal bore Varian NMR system.

- A $10 \mathrm{mT} 1.05 \mathrm{MHz}$ AMF was generated using a copper solenoid coil, diameter $3 \mathrm{~cm}$. Mice were placed inside the coil under anaesthesia and each hyperthermia session lasted 20 min.

- Post-heating histology was obtained.

Results Hyperthermia treatment caused a rise in temperature up to $60^{\circ} \mathrm{C}$ in vitro and a rise in tumour temperature of up to $4^{\circ} \mathrm{C}$ above body temperature in vivo (Abstract S89 Figure 1), detected by thermal imaging and with fibre optic probes. Iron was confirmed in the tumour using MR imaging and histology.

Discussion This study demonstrates the potential of SPION to act as an imaging agent and cancer therapy. SPION-labelled MSCs can be imaged in vivo in very low cell numbers. We have demonstrated that the application of an alternating magnetic field causes a temperature rise in these cells, both in vitro and in vivo. In the future, we will optimise SPION as an imaging and hyperthermia agent, for the targeted treatment of lung metastases.

Acknowledgements We are grateful for the support of the Medical Research Council.

\section{S90 HOMOCYSTINE LEVELS IN NON-SMALL CELL LUNG CANCER}

doi:10.1136/thx.2010.150938.41

J Maguire, V Kelly, C Smyth, M Ledson, M Walshaw. Liverpool Heart and Chest Hospital, Liverpool, UK

Plasma homocystine level is the most sensitive marker of sub-clinical folate deficiency. Elevated levels of homocystine have been associated with several pathologies, particularly vascular and cardiac disease. Patients with lung cancer are frequently elderly and may have insufficient intake of folate. Sub-clinical folate deficiency may lead to increased morbidity during chemotherapy and radiotherapy, and possibly reduced response rates to both treatment modalities. We have measured plasma homocystine levels in 460 patients with a diagnosis of non-small cell lung cancer who were referred to our lung cancer unit between 2005 and 2009. Two hundred and fifty-six patients were male, 204 were female. The median age was $71.22 \%$ of patients were PS $0-1,35 \%$ PS2 and 43\% PS3. The local laboratory reference range for homocystine is $\leq 15 \mu \mathrm{mol} / 1.43 \%$ of patients had elevated plasma levels of homocystine at diagnosis. In $35 \%$ the level was between 15.1 and $25 \mu \mathrm{mol} / \mathrm{l}$, and in $8 \%$ the homocystine level was greater than $25 \mu \mathrm{mol} / \mathrm{l}$. Patients with elevated plasma homocystine levels had inferior median and 1 year survival rates, both for stage III disease (464 vs 356 days ms, 1 year survival $65 \%$ vs $47 \%$ ) and stage IV disease (212 vs 190 days ms, 1 year 30\% vs $21 \%$ ). The finding of elevated homcystine levels in $43 \%$ of newly diagnosed patients with non-small cell lung cancer is of potentially profound significance, as this implies that there could be a possible therapeutic benefit from folate and B12 supplementation before and during treatment for this group of patients with non- small cell lung cancer.

\section{S91 THE ROLE OF THE VITAMIN D AXIS IN LUNG CANCER}

doi:10.1136/thx.2010.150938.42

${ }^{1}$ L A McGowan, ${ }^{2} \mathrm{~A}$ M Wood, ${ }^{2} \mathrm{P}$ Newby, ${ }^{3} \mathrm{~B}$ Naidu. ${ }^{1}$ University of Warwick, Warwick, UK; ${ }^{2}$ University of Birmingham, Birmingham, UK; ${ }^{3}$ Heart of England NHS Trust, Birmingham, UK

Introduction Vitamin D deficiency has been associated with many cancers, although little is known about its role in lung cancer. This is biologically plausible since vitamin $\mathrm{D}$ influences apoptosis and inhibits cell proliferation. Vitamin D receptor (VDR) polymorphisms have also been suggested to play a role in genetic susceptibility, their direction being consistent with inability to respond to vitamin $\mathrm{D}$. Finally, vitamin $\mathrm{D}$ binding protein (DBP) indirectly activates alveolar macrophages, a higher number of which within a tumour links to better prognosis in lung cancer.

Methods 37 patients with a diagnosis of lung cancer were studied in the first phase, together with 18 healthy controls. Circulating vitamin $\mathrm{D}$ level was measured at initial presentation by tandem mass spectrometry, and DBP by ELISA. Vitamin D and DBP level was compared between groups, and against other clinical features. VDR was quantified in normal lung and tumour tissue by immunohistochemistry, and compared between the two. In the second phase the lung cancer patients were genotyped for four functional SNPs in the vitamin D pathway, and their genotype frequencies compared to 484 healthy controls.

Results Vitamin D levels were lower in lung cancer than controls, after adjustment for season of collection $(21.8$ vs $15.5 \mathrm{ng} / \mathrm{ml}$, $\mathrm{p}=0.018$ ), as was DBP (28.2 vs $51.7 \mathrm{mg} / \mathrm{dl}$ ). DBP was higher in women $(p=0.001)$ and correlated directly with vitamin $D(r=0.446$, $\mathrm{p}=0.013$ ). VDR was expressed primarily in bronchial epithelium, and to a lesser extent in pneumocytes, but was generally expressed

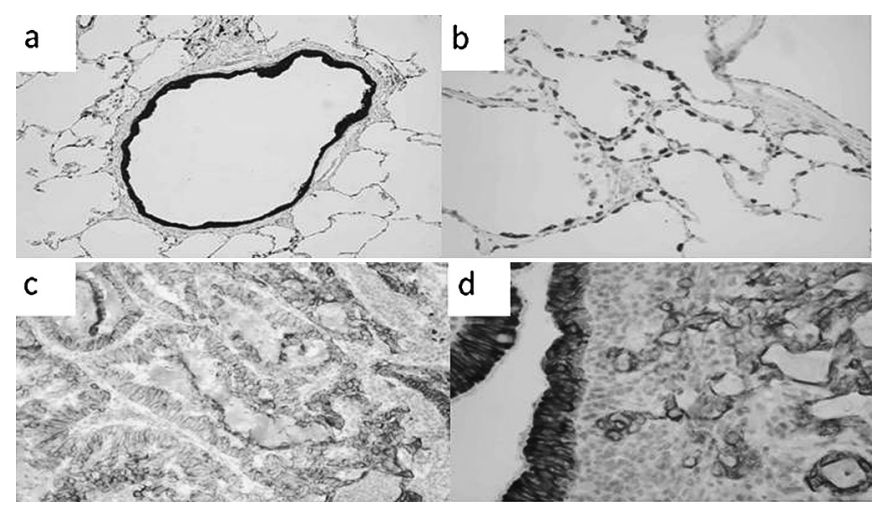

Abstract S91 Figure 1 VDR stains brown in (A) bronchial epithelium (B) less strongly in pneumocytes and (C\&D) adenocarcinoma. Tissue in all bar (D) is from the same individual. (D) shows both epithelium and cancer tissue. Similar results were seen in two patients with squamous carcinomas. 